\title{
Filosofía ambiental de campo: ecología y ética en las redes LTER-Chile e ILTER
}

\author{
Field environmental philosophy: ecology and ethics in LTSER-Chile and ILTER networks
}

\author{
Ricardo Rozzi $^{\text {a,b,c* }}$, Francisca Massardo ${ }^{\text {a,b }}$, Tamara Contador ${ }^{\text {a,b }}$, Ramiro D Crego ${ }^{\text {b,c }}$, Manuela Méndez ${ }^{\text {b,d }}$, \\ Rajan Rijal c, Lohengrin A Cavieres ${ }^{\text {b,d }}$, Jaime E Jiménez ${ }^{\text {a,b,c }}$ \\ *Autor de correspondencia: ${ }^{a}$ Universidad de Magallanes, Parque Etnobotánico Omora, Puerto Williams, Chile, rozzi@unt.edu

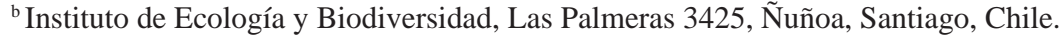 \\ ${ }^{c}$ University of North Texas, Sub-Antarctic Biocultural Conservation Program, Denton, TX, USA. \\ ${ }^{\text {d} U n i v e r s i d a d ~ d e ~ C o n c e p c i o ́ n, ~ c a s i l l a ~ 160-C, ~ C o n c e p c i o ́ n, ~ C h i l e . ~}$
}

\begin{abstract}
SUMMARY
The long-term socio-ecological research (LTSER) study sites should integrate theoretical and applied research to effectively confront global socio-environmental change. For a socio-ecological integration beyond the socio-economic approach that prevails today in LTSER networks worldwide, in the southernmost site of the LTSER-Chile network (Omora Park, $55^{\circ} \mathrm{S}$ ) we have developed the Field Environmental Philosophy (FEP) methodological approach. This paper presents a methodological framework and applications of FEP at Omora Park that combine ecological and philosophical research in education and biocultural conservation programs, including ecotourism. Through in situ and in tempo work with educators, policy makers and other stakeholders, researchers not only investigate the relations between society and ecosystems, but also transform them. This ecological and ethical understanding orient educational and governance processes toward ecological, socio-cultural and economic sustainability. The experiences of FEP demonstrate how this methodology transforms socio-ecological relationships by: (i) changes in language that generate in society a "change of lenses" to appreciate values and the symbolic-linguistic reality, usually studied by philosophers; this change determines, in turn, (ii) transformations in the ways in which society understands and value the biophysical reality of ecosystems, usually studied by ecologists. Globally, the FEP provides a methodology that contributes to orient global society towards ways that better understand and value biodiversity, favoring the sustainability of life.
\end{abstract}

Key words: biocultural conservation, environmental ethics, metaphors, Omora Park, socio-ecological.

\section{RESUMEN}

Los sitios de estudios socio-ecológicos a largo plazo (LTSER) debieran integrar investigación teórica y aplicada para confrontar efectivamente el cambio socio-ambiental global. Para lograr una integración socio-ecológica que vaya más allá del enfoque socioeconómico que prevalece hoy en las redes LTSER a nivel mundial, en el sitio más austral de la Red LTSER-Chile (Parque Omora, $55^{\circ} \mathrm{S}$ ) se ha desarrollado la Filosofía Ambiental de Campo (FILAC). Este artículo presenta el marco metodológico y casos de aplicación de la FILAC en el Parque Omora que combinan investigaciones ecológicas y filosóficas con educación y conservación biocultural, incluyendo el ecoturismo. A través de este trabajo, los investigadores no sólo estudian las relaciones entre la sociedad y los ecosistemas, sino que también las transforman. Esta comprensión ecológica y ética orienta procesos educativos y de gobernanza hacia la sustentabilidad ecológica, socio-cultural y económica. Las experiencias de la FILAC demuestran cómo esta metodología transforma las relaciones socio-ecológicas a través de: (i) cambios en el lenguaje, que provocan en la sociedad un “cambio de lentes” de la realidad simbólico-lingüística y valórica, habitualmente estudiada por filósofos; estos cambios determinan, a su vez, (ii) modificaciones en los modos en que la sociedad comprende y valora la realidad biofísica de los ecosistemas, habitualmente estudiada por ecólogos. A nivel mundial, la FILAC aporta una metodología que contribuye a transformar la forma prevaleciente en que la sociedad global comprende, valora y se relaciona con los ecosistemas hacia formas que favorecen la sustentabilidad de la vida.

Palabras clave: conservación biocultural, ética ambiental, metáforas, Parque Omora, socio-ecológico.

Una ética de la tierra refleja la existencia de una conciencia ecológica y, a su vez, esta conciencia refleja una convicción de responsabilidad individual por la salud de la tierra. La salud es la capacidad de la tierra para autorregenerarse. La conservación es nuestro esfuerzo por entender y conservar esta capacidad (Aldo Leopold 1949; énfasis añadido). 


\section{INTRODUCCIÓN: UN PROGRAMA SOCIO- ECOLÓGICO TRANSDICIPLINARIO EN LA RED LTSER-CHILE}

Para confrontar el vertiginoso cambio socio-ambiental global, los sitios de estudios socio-ecológicos a largo plazo debieran cumplir una doble función: entender los ecosistemas y su capacidad para mantener la vida, humana y no-humana, y conservar esta capacidad o salud de los ecosistemas. Los ecosistemas incluyen a los seres humanos y al conjunto de los seres vivos. Para conservar la capacidad de los ecosistemas para sustentar la vida es fundamental integrar los saberes teóricos y prácticos. La necesidad de integrar estos saberes (frecuentemente subvalorada por políticas de desarrollo científico que se enfocan en estudios y publicaciones teóricas), ha sido enfatizada por pedagogos como Paulo Freire (1970), por ecólogos como Aldo Leopold (1949), y en la propuesta de conservación biocultural desarrollada en el Parque Etnobotánico Omora, el sitio más austral de la Red Chilena de Estudios SocioEcológicos a Largo Plazo (LTSER-Chile) ${ }^{1}$ (Rozzi et al. 2008, 2010a, 2012).

Bajo el enfoque de la conservación biocultural, los sitios de la red LTSER-Chile no constituyen meros observatorios ecológicos que se limitan a investigar o monitorear los ecosistemas, sino que desarrollan (o debieran desarrollar) programas socio-ecológicos transdisciplinarios. A partir de la analogía leopoldiana entre la conservación y la salud, proponemos metafóricamente que cada sitio de la Red LTSER-Chile debe cumplir una función doble, similar a un laboratorio natural y a un hospital natural. El hospital no es sólo un laboratorio donde se estudian los pacientes, sino que también es un lugar donde se los trata, procurando mantener o devolver su salud. El resultado último es la salud de los pacientes; más que generar investigaciones y publicaciones. De manera equivalente, bajo la pedagogía freiriana, la escuela es un lugar donde se establecen prácticas de aprendizaje recíproco entre estudiantes y maestros; no sólo se informa a los estudiantes (Freire 1970). Por lo tanto, un hospital y una escuela no pueden ser concebidos como instituciones que se visitan ocasionalmente para realizar estudios de los pacientes o estudiantes, sino que requieren una atención diaria in situ. Análogamente, si los sitios de la Red LTSER-Chile aspiran a una integración teórico-práctica con implicancias para la conservación y la toma de decisiones medioambientales, entonces estos sitios requieren un funcionamiento diario in situ, además de programas de investigación y publicaciones acerca de sus ecosistemas. Para alcanzar esta integración teóricopráctica transdisciplinaria en los sitios LTSER-Chile, se requieren innovaciones institucionales y en las políticas de financiamiento y evaluación de las ciencias ecológicas en el país, como las que han inaugurado la Iniciativa Científica Milenio del Ministerio de Economía y el Programa de

LTSER, sigla en inglés de Long-Term Socio-Ecological Research.
Financiamiento Basal para Centros de Excelencia Científica y Tecnológica de CONICYT en Chile.

En el Parque Omora $\left(55^{\circ} \mathrm{S}, 67^{\circ} \mathrm{O}\right)$ hemos abordado este desafío institucional desarrollando un programa transdisciplinario que requiere interacciones continuas con múltiples instituciones gubernamentales, privadas, educativas y de la comunidad. Trabajamos tanto en investigación como en conservación a largo plazo dentro de sistemas socio-ecológicos en la Reserva de Biosfera Cabo de Hornos: el equipo observa y estudia a la vez que participa y transforma trayectorias socio-ecológicas. Para esta integración teórico-práctica, el equipo del Parque Omora ha confrontado además del desafío institucional, un desafío metodológico para lograr una integración socio-ecológica que vaya más allá del enfoque utilitarista y economicista que prevalece hoy en las redes LTSER a nivel mundial, donde lo socioecológico se reduce frecuentemente a socio-económico (Rozzi et al. 2012). Para superar los problemas socio-ambientales actuales es esencial incluir no sólo valores económicos sino también éticos, pero esto presenta una limitación crítica para la inclusión de la ética en la conservación: la falta de metodologías para guiar el trabajo transdisciplinario de ecólogos, filósofos y otros participantes (Poole et al. 2013). Para resolver esta limitación metodológica, en el Parque Omora hemos propuesto la Filosofía Ambiental de Campo (FILAC) (Rozzi et al. 2005, 2008, 2012).

Este artículo presenta un marco filosófico y casos de aplicación del enfoque metodológico de la FILAC con los objetivos de contribuir a una mejor traducción de conceptos valóricos e incorporación de prácticas éticas en la conservación. Estas prácticas procuran superar la compartimentalización disciplinaria e institucional de la educación y la política (Oeschlaeger y Rozzi 1996) y enfatizar una conservación in situ participativa con la comunidad en los procesos de aprendizaje, descubrimiento y cuidado de la salud de los ecosistemas.

\section{MÉTODOS Y RESULTADOS}

Filosofía Ambiental de Campo (FILAC) y ética biocultural. La FILAC no es una metodología meramente teórica, sino que se sustenta en una ética biocultural que propone límites a la acción de la política económica neo-liberal imperante. La ética biocultural extiende la comunidad moral más allá de quienes se benefician con el libre mercado, para incluir a la mayoría de las poblaciones humanas marginadas y oprimidas, y más allá de la especie humana para incluir a todos los seres con que co-habitamos en la biosfera (Rozzi 2012). En suma, la ética biocultural demanda una justicia ecosocial que considera la comunidad de cohabitantes humanos y no-humanos.

El sentido filosófico del término compuesto bio-cultural integra dos niveles de la realidad: el biofísico (común a los seres humanos y otros seres vivos) y el cultural o simbólicolingüístico que se refiere a cómo los seres humanos (incluyendo los científicos) representamos a los seres vivos y nos 
relacionamos con ellos. Habitualmente, los ecólogos centran su investigación en la realidad biofísica y los filósofos en el examen de la realidad cultural y del lenguaje. La ética biocultural vincula, en cambio, los niveles biofísico y cultural. Para ello se centra en las interrelaciones entre los hábitats (dónde vivimos, usualmente estudiados por los ecólogos) y los hábitos (cómo vivimos, usualmente estudiado por filósofos y otras disciplinas sociales) (Rozzi et al. 2008).

La FILAC provee un marco metodológico que ha permitido a estudiantes de postgrado vinculados al Parque Omora combinar investigaciones ecológicas y filosóficas en una práctica teórica y aplicada de educación y conservación biocultural. Una forma de contribuir a la implementación de la conservación biocultural es el ecoturismo.

Ética biocultural y ecoturismo. El ecoturismo en sentido estricto requiere una comprensión de los vínculos entre los hábitats de una región y los hábitos de vida de sus habitantes. Hoy, el turismo de naturaleza es a menudo masivo y niega estos vínculos (Acevedo 2006). Consecuentemente, genera una homogeneización biocultural y una injusticia ecosocial en lugares emblemáticos como Costa Rica, las Islas Galápagos y Torres del Paine. Es imprescindible corregir las ambigüedades del "marketing verde" del turismo de naturaleza y prestar mayor atención a la diversidad biocultural para poner en práctica un ecoturismo genuino. La ética biocultural considera al ecoturismo como una oportunidad única para reconectar a los ciudadanos de la sociedad global con la diversidad de la vida humana y nohumana. Eco-turismo es una palabra compuesta por la raíz griega oikos, que significa hogar o hábitat, y el término francés tour que significa viaje. Basado en el fundamento de integración entre hábitos humanos y sus relaciones con las comunidades de co-habitantes y los hábitats donde tienen lugar, hemos propuesto la siguiente definición de ecoturismo desde el marco conceptual de la ética biocultural: ecoturismo es un tour o viaje para apreciar y compartir los hábitos de vida de co-habitantes humanos y no-humanos en sus oikos o hábitats locales, que conlleva beneficios para quienes visitan y quienes son visitados (Rozzi et al. 2010b).

La FILAC concibe al ecoturismo como una actividad que debe ser guiada con un sentido ecológico y ético, para orientar al visitante para conocer, comprender, disfrutar y respetar la diversidad biocultural y reporta beneficios para la comunidad hospedera, humana y no-humana. El ecoturismo es especialmente relevante para que sectores más amplios de la sociedad conozcan y valoren hábitats, hábitos culturales y comunidades biológicas poco conocidas, que permanecen "invisibles" para la mayoría de la ciudadanía. Más invisible aún permanecen las relaciones ecológicas y éticas que la sociedad mantiene y debiera mantener con esta biodiversidad.

Ciclo metodológico de la Filosofía Ambiental de Campo (FILAC). La FILAC contribuye a visibilizar y comprender las relaciones éticas y ecológicas con la diversidad biológica y cultural. Para ello integra las ciencias ecológicas y la ética ambiental a través de un ciclo de cuatro pasos interrelacionados entre sí: i) investigación interdisciplinaria ecológica y filosófica, ii) composición de metáforas y comunicación a través de relatos simples, iii) diseño de experiencias de campo guiadas con un sentido ecológico y ético, y iv) habilitación de áreas y/o prácticas de conservación in situ (figura 1).

En el paso 1 los estudiantes realizan investigaciones ecológicas, etnoecológicas y filosóficas, incluyendo el análisis de tres tipos de textos: (a) trabajos fundacionales de la ética ambiental (e.g., Leopold 1949, Boff 1995); (b) textos que acuñan la terminología para referirnos a la biodiversidad en los lenguajes de diferentes disciplinas, instituciones y grupos socioculturales, que implican una diversidad de valores y percepciones acerca de la diversidad bio-cultural, formas de conocimiento y prácticas ecológicas (e.g., Schüttler et al. 2011, Rozzi y Jiménez 2014), y (c) textos que elaboran la propuesta de la ética biocultural y la filosofía ambiental de campo en el Parque Etnobotánico Omora y la Red LTSER-Chile (e.g., Rozzi et al. 2005, 2012). De esta manera, los estudiantes realizan análisis comparativos entre los conocimientos de los diversos grupos socioculturales, sus modos de comprensión y de valoración, identificando así similitudes y diferencias entre los varios modos de conocer, valorar y convivir con la diversidad biocultural. Esta investigación les permite reconocer causas más específicas de algunos problemas ambientales, junto con oportunidades para resolverlos.

El paso 2 tiene dos objetivos fundamentales: a) integrar la investigación ecológica y filosófica a través de un pensamiento analógico y de un trabajo poético que conduce a una síntesis conceptual de hechos, valores y acciones de educación y/o conservación biocultural; b) generar relatos e imágenes mentales para comunicar al público general los principales resultados obtenidos en la investigación (paso 1).

El paso 3 estimula la percepción y valoración de la biodiversidad mediante "encuentros directos" con la comunidad de diversos cohabitantes en sus hábitats. Para ello, los estudiantes diseñan actividades de educación ambiental y ecoturismo que permiten compartir los resultados obtenidos de la investigación (paso 1) y los conceptos propuestos en los relatos simples y metáforas compuestas (paso 2) con miembros de todas las edades de la comunidad local y visitantes de manera experiencial en terreno.

El paso 4 consiste en la habilitación temática y/o física de estaciones interpretativas o áreas para la protección de los habitantes, sus hábitos y hábitats (hábitats nativos, especies e interacciones ecológicas) en los cuales se realizaron los estudios ecológicos. Para los visitantes, la implementación de estas áreas de conservación biocultural in situ posibilita experimentar la diversidad biocultural a través de encuentros directos con sus co-habitantes; para los estudiantes, fomenta la responsabilidad ecológica y ética y la proactividad para conservar la diversidad biocultural. 


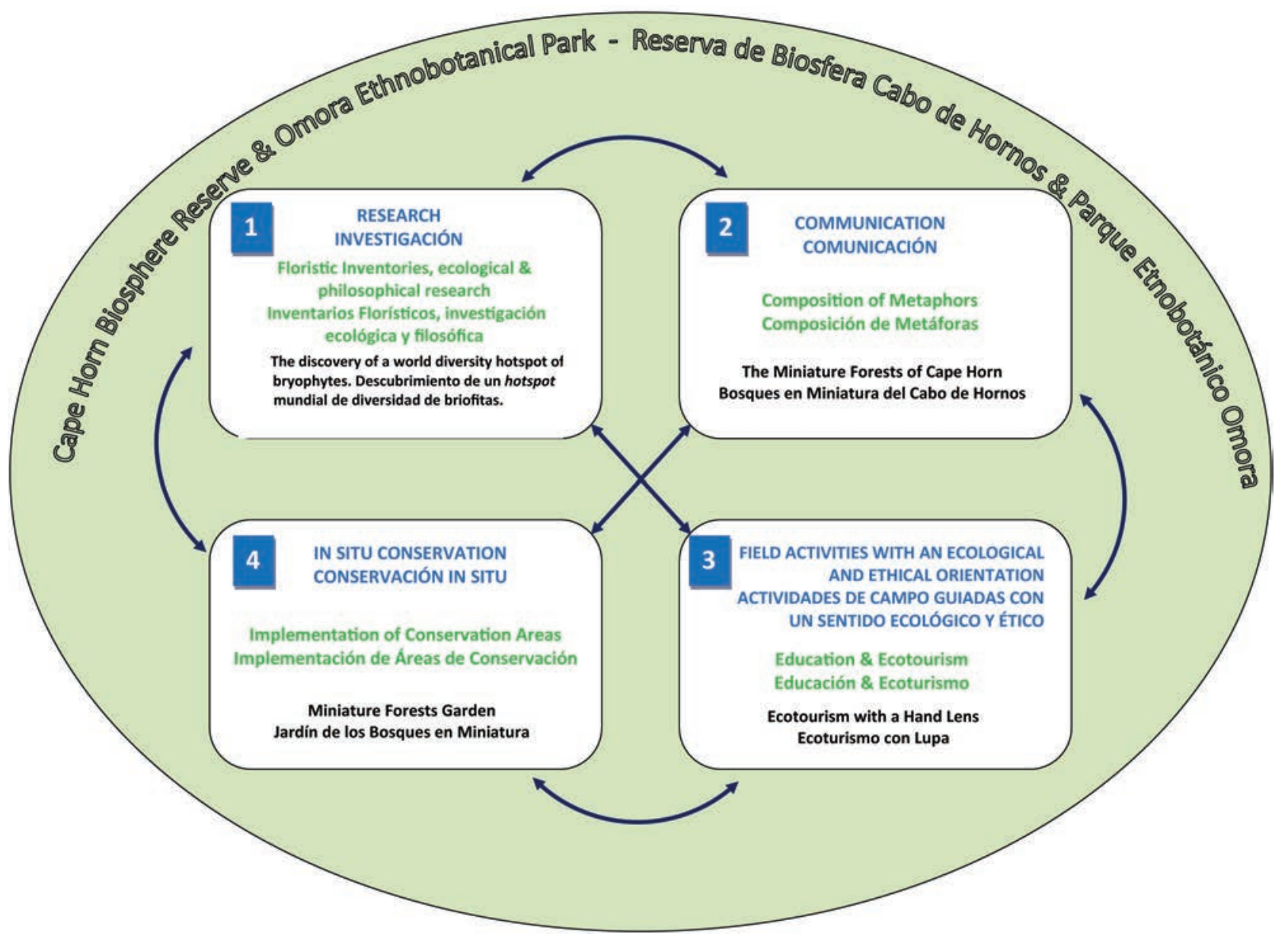

Figura 1. Modelo metodológico de la filosofía ambiental de campo: ciclo de cuatro pasos para integrar las ciencias ecológicas y la ética ambiental en la conservación y el ecoturismo biocultural, ilustrado con el ejemplo de "Ecoturismo con lupa". En cada paso del ciclo (azul) se señala el método (verde) y los resultados (negro) para la conservación de la flora no-vascular en la Reserva de Biosfera Cabo de Hornos. Las flechas y líneas indican que las interacciones entre los cuatro pasos son multidireccionales.

Methodological model of Field Environmental Philosophy: four-step cycle to integrate ecological sciences and environmental ethics into biocultural conservation and ecotourism, showed with the example of "Ecotourism with a Hand-Lens." Each cycle's step (blue) is illustrated according to the method (green), and the results (black) toward conserving the non-vascular flora in the Cape Horn Biosphere Reserve. Arrows and lines indicate that interactions among the four steps are multidirectional.

Este ciclo se ha definido a posteriori analizando las experiencias efectivas del programa de investigación, educación y conservación biocultural a largo plazo del Parque Omora, asociadas al desarrollo de tesis de postgrado de estudiantes de la Universidad de Magallanes (UMAG) y otras universidades chilenas e internacionales. A partir del 2008, el ciclo de la FILAC se ha adoptado como una metodología estructurada para el diseño de tesis y mallas curriculares de estudiantes que están generando innovadoras actividades educativas, de conservación y de ecoturismo, tales como el "ecoturismo con lupa” (Rozzi et al. 2005, 2008) y “ojo bucea con ojo” (Ojeda 2013). Un aspecto metodológico esencial en el desarrollo de estas tesis ha sido la co-tutoría entre ecólogos, filósofos y/o biólogos de la conservación.

Para ilustrar concisamente la metodología de la FILAC, se presentan dos casos de tesis recientes desarrolladas en el
Parque Etnobotánico Omora por Manuela Méndez (2013, Universidad de Concepción e Instituto de Ecología y Biodiversidad [IEB]) y Tamara Contador (2011, University of North Texas [UNT] y UMAG). Los estudiantes han desarrollado su trabajo en conjunto con los investigadores y diversos miembros de la comunidad, quienes participan en la generación de nuevos descubrimientos científicos. Así, los estudios a largo plazo generados en el Parque Omora no sólo contribuyen a generar datos científicos relevantes para la academia, sino que los datos son generados en conjunto con la comunidad y compartidos con tomadores de decisiones y autoridades de gobierno. Esto permite cultivar una ética que refleja una responsabilidad individual por la salud de la tierra, a través de un entendimiento colectivo sobre la necesidad de conservar su capacidad para autorregenerarse en el corto y largo plazo. 


\section{CASO 1: JARDINERAS SUBANTÁRTICAS} ALTOANDINAS

Paso 1. Investigación interdisciplinaria: diversidad y microhábitats de la flora altoandina subantártica. En la Reserva de Biosfera Cabo de Hornos, los ambientes altoandinos se encuentran por sobre una línea claramente distinguible a la distancia que separa los bosques de las laderas de las cumbres de los cerros: el límite arbóreo. Para la investigación del dominio simbólico-lingüístico se analizó el lenguaje referido a las comunidades altoandinas en la obra de Edmundo Pisano, botánico y explorador magallánico. Pese a que Pisano conocía perfectamente la flora altoandina de Magallanes, se refiere a este hábitat montañoso como "desierto andino" (Pisano 1980), evocando una imagen mental que sugiere que las cumbres de las montañas subantárticas constituyen "desiertos"; es decir, un paisaje desprovisto de vegetación.

Para la investigación del dominio biofísico se examinaron los patrones de diversidad de musgos y plantas vasculares en los hábitats altoandinos aledaños al Parque Omora (5458' S, 67³8' O). En dos laderas, cada una con 50 pequeñas "islas de vegetación" asociadas a plantas en cojín de Bolax gummifera (Lam.) Spreng. se registraron 52 especies: 34 musgos y 18 plantas vasculares (Méndez et al. 2013) (figura 2). Este estudio demostró cuantitativamente una alta riqueza de especies vegetales presentes en este "desierto", y complementó los trabajos cualitativos desarrollados por Pisano (1980). Pese a su gran conocimiento florístico, Pisano inadvertidamente traiciona su comprensión del dominio biofísico al utilizar la representación visual de desierto andino en el dominio simbólico-lingüístico. Desde el punto de vista de la ética, esta discrepancia entre ambos dominios es problemática puesto que, como afirma Leopold en su "Ética de la tierra", "sólo podemos actuar éticamente en relación con aquello que podemos ver, sentir, comprender, amar o de algún modo tener fe” (Leopold 1949).

Paso 2. Comunicación y composición de metáforas: jardineras subantárticas altoandinas. Para reparar la incongruencia entre los dominios biofísico y simbólico-lingüístico, se trabajó en la generación de una imagen mental alternativa a “desierto andino". Las plantas en cojín, como B. gummifera, constituyen una de las formas de vida más características de los ambientes de alta montaña y pueden generar microhábitats que favorecen el establecimiento de otras plantas dentro del cojín, más que sobre el suelo adyacente. Esto sugiere que las especies en cojín actuarían como facilitadoras (Méndez et al. 2013). Metafóricamente, las plantas en cojín actúan como "jardineras", formando islas de "jardines subantárticos” que proporcionan un sustrato orgánico más estable y abrigado que permite el crecimiento de musgos y plantas con flores. Basado en esta comprensión botánica y ecológica, el trabajo poético cambia nuestra representación mental desde un "desierto andino" hacia el concepto metafórico de "jardineras subantárticas altoandinas".
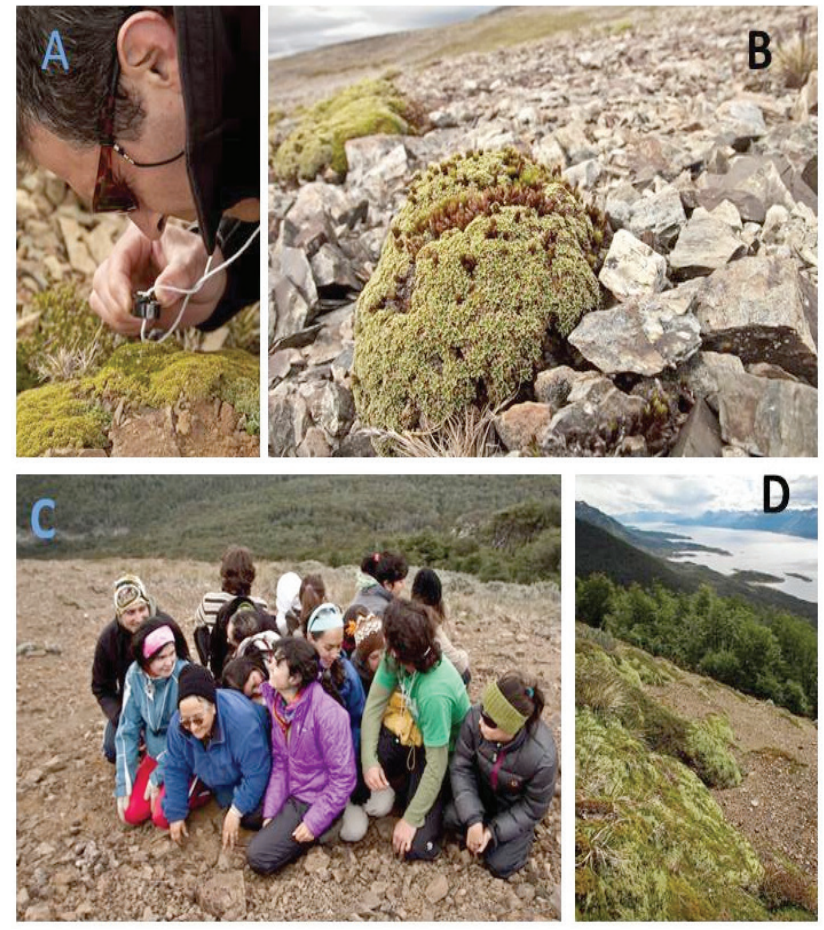

Figura 2. Ecoturismo con lupa en las jardineras subantárticas altoandinas. A) Investigación interdisciplinaria de la diversidad florística en las plantas en cojín del altoandino en el cerro Bandera, Isla Navarino. B) Comunidad vegetal asociada a cojines de Bolax gummifera, metafóricamente denominada "jardinera subantártica altoandina”. C) Actividad de campo ecológica y éticamente guiada "co-habitando como plantas altoandinas". D) Conservación in situ de comunidades vegetales asociadas a plantas en cojín en el circuito interpretativo del Parque Omora Sendero de Chile, Cerro Bandera. Fotografías Gonzalo Arriagada.

Ecotourism with a hand-lens in the high-Andean subAntarctic gardeners. A) Interdisciplinary research of floristic diversity in the high Andean cushion plants at Bandera Mountain, Navarino Island. B) Plant community associated to Bolax gummifera, metaphorically called “ high-Andean sub-Antarctic gardener.” C) “Co-inhabiting like high-Andean plants," field activity guided with an ecological and ethical orientation. D) In situ conservation of plant communities associated to cushion plants in the interpretive trail Omora Park - Sendero de Chile, Bandera Mountain. Photographs Gonzalo Arriagada.

Paso 3. Actividades de campo ecológica y éticamente guiadas: co-habitando como plantas altoandinas. Para los visitantes de la zona altoandina la experiencia comienza al cruzar el límite arbóreo, formado por árboles achaparrados y antiguos. Aquí se invita a los visitantes a sentir el viento, observar a los cohabitantes de este hábitat, disfrutar de la vista aérea y preguntarse: ¿cómo logran las plantas en cojín vivir en ese ambiente tan inhóspito, expuesto a la nieve, al viento, a sustratos inestables? Para responder esta pregunta, se diseñó una actividad de ecoturismo en las jardineras subantárticas de tal manera que los visitantes experimentan las condiciones del altoandino contrastando el crecimiento individual más separado en el bosque y otros hábitats pro- 
tegidos. A partir de esta experiencia, se puede reflexionar sobre una implicancia ética: en la naturaleza, tal como en la sociedad humana, no sólo existe competencia sino también colaboración para cohabitar y florecer incluso en ambientes rigurosos como las cumbres subantárticas.

Paso 4. Conservación in situ: estación de interpretación ecoturística en el tramo más austral del Sendero de Chile. Chile es un país montañoso que atrae a visitantes de todo el mundo para practicar montañismo, escalada y caminatas. Para la nueva actividad de ecoturismo en las jardineras subantárticas altoandinas, se han diseñado estaciones interpretativas en sectores del Sendero de Chile aledaños al Parque Omora. Estas estaciones orientan al visitante, lo invitan a acercarse al suelo con una lupa, observar y proteger la diversidad de pequeñas plantas y animales que cohabitan en las montañas subantárticas del Cabo de Hornos.

\section{CASO 2: SUMERGIDOS CON LUPA}

Paso 1. El colibrí omora y los invertebrados dulceacuícolas de Cabo de Hornos. En la ecorregión subantártica de Magallanes habita una gran diversidad de invertebrados dulceacuícolas que ayudan a mantener la calidad del agua y que han sido poco estudiados (Contador et al. 2012). Para la investigación del dominio simbólico-lingüístico, se analizó la historia yagán del picaflor omora (Sephanoides sephaniodes). De acuerdo a la cosmogonía yagán, al crear los cauces de agua, omora protege a las comunidades de animales y plantas y enfatiza la importancia de la integridad de las comunidades biológicas para el mantenimiento de las fuentes de agua para los seres humanos y no-humanos. Esta cosmogonía amerindia converge con la comprensión científica actual de servicios ecosistémicos (Rozzi y Jiménez 2014).

Para la investigación del dominio biofísico se estudió la diversidad y ciclos de vida de los invertebrados del río Róbalo, que cruza el Parque Omora desde las cumbres hasta su desembocadura en el canal Beagle. Tricópteros y dípteros son notables por exhibir características singulares en sus historias de vida (Contador 2011). Los tricópteros construyen una "madriguera" (figura 3), hábito que contribuye a comprender estos insectos como sujetos activos. Esta comprensión supera la dicotomía cartesiana objeto/ sujeto que domina el pensamiento moderno, para el cual el único sujeto activo que posee valor en sí mismo es el ser humano. Así, bajo la ética biocultural, tricópteros y humanos poseen un ethos en el sentido de hábitat y hábitos (e.g., constructor de hábitat) y todos lo seres vivos, humanos y no-humanos, son considerados como sujetos co-habitantes que tienen valor intrínseco y dignidad.

El díptero Gigantodax rufescens (Simuliidae) presentó un ciclo de vida muy sensible a cambios de temperatura. En respuesta al gradiente térmico altitudinal del río Róbalo (586 m - 0 m), su ciclo de vida varía de multivoltino a univoltino, i.e., desde varias a solo una generación por año

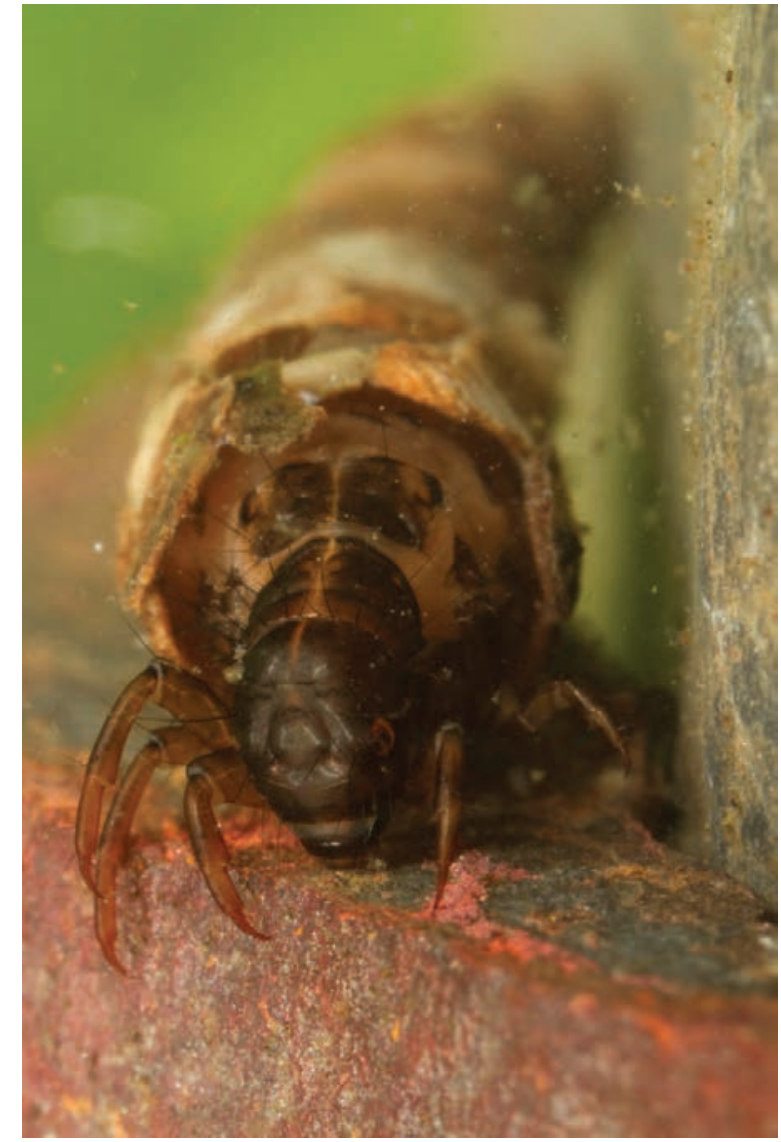

Figura 3. Larva de tricóptero (Monocosmoecus sp.) que habita bajo el agua en el río Róbalo dentro de su cocón o "madriguera" (Gr. ethos, origen etimológico de la palabra ética), fabricada con sus glándulas productoras de seda a la que adhieren detritus vegetales, arena y piedras pequeñas. Fotografía Gonzalo Arriagada.

Trichoptera or caddisfly larva living under water in the Robalo River inside its case or "den" (=Gr. ethos, etymological origin of the word ethics), built with its salivary glands that excrete silk, material to which they adhere small pieces of twig, sand, and small fragments of rock, or aquatic plants. Photograph Gonzalo Arriagada.

(Contador 2011, Contador et al. 2014). Esta característica otorga a $G$. rufescens un valor instrumental para evaluar modelos predictivos del impacto del cambio climático sobre comunidades dulceacuícolas.

Paso 2. El río como comunidad de vida. Para integrar los resultados obtenidos en los dominios biofísico y simbólico-lingüístico, se trabajó poéticamente en la generación de una imagen mental alternativa a la de "recurso natural". Una implicancia ética central de los resultados del paso 1 es que ambos insectos poseen valor instrumental e intrínseco. Además, son parte de una comunidad de cohabitantes cuyos hábitos de vida y hábitats se interrelacionan. La historia yagán de omora, la construcción de microhábitats del tricóptero y los cambios de ciclo de vida del díptero evocan una comprensión del río como "Comunidad de Vida". 
Paso 3. Sumergiéndose con lupa. Esta actividad invita a personas de todas las edades a descubrir y valorar la "Comunidad de Vida” del río a través de encuentros directos con los invertebrados dulceacuícolas. Los visitantes experimentan una transformación ética a través de tres pasos: (i) reconocimiento de macro y micro-hábitats dentro y fuera del río, (ii) encuentros directos con los habitantes sumergidos y (iii) respeto y valoración por los habitantes, sus hábitos y hábitats. Los visitantes recogen una roca del río recordando el lugar exacto de donde la tomaron y la depositan en una bandeja con agua para observar los desplazamientos de sus habitantes. Con una lupa los observan e identifican y devuelven la roca al mismo lugar dónde estaba. Los visitantes descubren que las rocas sumergidas son la "casa" o microhábitat de una gran variedad de habitantes sumergidos. Regresarla a su lugar estimula un sentido de responsabilidad y una experiencia de transformación ética, valorando la casa de otros seres no-humanos, respetando sus hábitats y sus hábitos.

Paso 4. Circuito de los habitantes sumergidos en los ríos del Cabo de Hornos. Para desarrollar nuevas formas de turismo sustentable en la región de Magallanes y promover la conservación in situ de los insectos dulceacuícolas, se diseñó y construyó el circuito interpretativo de los Habitantes Sumergidos en los ríos del Cabo de Hornos en el Parque Omora. Este circuito introduce un nuevo tipo de ecoturismo en Chile y Sudamérica enfocado en la conservación de los insectos dulceacuícolas. A través de cinco estaciones interpretativas, el circuito contribuye a promover la observación y la valoración la biodiversidad asociada al río Róbalo, el cual provee de agua bebestible a Puerto Williams, de sus hábitos y hábitats.

\section{DISCUSIÓN Y CONCLUSIONES: INTEGRACIÓN DE LAS CIENCIAS ECOLÓGICAS Y LA ÉTICA AMBIENTAL EN LTSER-CHILE E ILTER}

A través del trabajo in situ e in tempo con educadores, tomadores de decisiones y otros actores, los investigadores asociados a un sitio de la Red LTSER-Chile no solo estudian las relaciones entre la sociedad y los ecosistemas, sino que también las transforman. La comprensión ecológica y axiológica (valórica) orienta los procesos educativos y de gobernanza hacia la sustentabilidad de la vida. Las experiencias de la FILAC demuestran cómo las transformaciones de las relaciones socio-ecológicas se impulsan con cambios en el lenguaje. Los dos ejemplos de tesis expuestos en este trabajo muestran cómo la comunidad educativa de Puerto Williams y la sociedad regional transita: 1) desde una visión de un "desierto altoandino" hacia la percepción de una rica flora de pequeñas plantas vasculares y no-vasculares que crecen en torno a plantas en cojín, que actúan como "jardineras subantárticas”; 2) desde una visión del río como un "flujo de agua" hacia una visualización de centenares de pequeños animalitos, plantitas y algas macro y microscópicas que constituyen una "comunidad de cohabitantes" o "comunidad de vida".

Estos cambios de nombre provocan un "cambio de lentes simbólico-lingüísticos” en la sociedad que, a su vez, cambia sus modos de comprender y valorar la realidad biofísica de los ecosistemas. A nivel gubernamental, la metodología de la FILAC ha estimulado cambios de nombre en la comuna, de Navarino a Cabo de Hornos (2001), la ecorregión, de Patagonia a Subantártica de Magallanes (año 2002), y la caleta, desde Hacienda Róbalo a Parque Etnobotánico Omora (2000). Estos cambios de nombre han estimulado, a su vez, un cambio en la gobernanza hacia la creación de la Reserva de Biosfera Cabo de Hornos por parte del Estado de Chile y la UNESCO (2005), el reconocimiento de una identidad de la Región de Magallanes como región antártica y subantártica, y la creación de un parque público-privado. El Parque Omora re-valora la lengua yagán para nombrar a los lugares, los ecosistemas y la biodiversidad subantártica, protege la cuenca hidrográfica del río Róbalo, ha implementado un "laboratorio natural" y una "escuela natural" para la investigación, la educación y la conservación biocultural, y ha cofundado la red LTSER Chile (2008).

A nivel mundial, la FILAC aporta un cambio de lenguaje que influye en la forma prevaleciente en la cual la sociedad global se relaciona con los ecosistemas. El lenguaje forjado por el utilitarismo y el liberalismo económico propuesto hace dos siglos por Adam Smith (1794) y Stuart Mill (1848) se ha globalizado. Estos autores consideraron a la tierra como un elemento de producción y capital. Bajo esta visión utilitarista, hoy globalizada con el neoliberalismo económico, el único valor de la tierra es su capacidad de producción y hoy, es común utilizar la expresión "recursos naturales" para referirse a la biodiversidad. En esta visión, la "tierra" y la biodiversidad son bienes; meros objetos a ser dominados, conquistados y explotados por los sujetos humanos. Esta concepción impide concebir a la naturaleza como una realidad viva, como una comunidad de co-habitantes con sus propias subjetividades.

El lenguaje utilitarista reduce la biodiversidad a meros "recursos naturales" y, por tanto, sometida a los deseos de la explotación de la sociedad humana gobernada por el libre mercado (esto es, libre de "frenos" a los intereses del mercado y de los sujetos humanos que participan en él). Embebida en esta ideología de cosificación y mercantilización de la biodiversidad, la conservación contemporánea procura una valoración bajo estimaciones monetarias y de servicios ecosistémicos (Costanza et al. 2014). La emergencia de este concepto ilustra cómo el lenguaje utilitarista sigue constituyendo una racionalidad omnipresente para concebir a la biodiversidad como un recurso esencialmente económico. El lenguaje utilitarista, con sus formas de nombrar, concebir y valorar la biodiversidad, influye en las formas en que la sociedad global administra y mercantiliza hoy a los seres vivos y los ecosistemas.

Bajo la evidencia ofrecida por las ciencias ecológicas contemporáneas, sin embargo, la concepción utilitarista de 
la biodiversidad como mero elemento de producción y capital es epistemológicamente reduccionista y éticamente injusta. Las ciencias muestran que la biodiversidad está formada por comunidades de seres vivos que son parientes evolutivos de la especie humana, y co-habitan con los seres humanos en los ecosistemas locales y la biosfera como un todo. La visión de que todos los seres están al servicio de una sola especie no es sustentable ecológicamente (Naeem 2013), ni tampoco es aceptable éticamente (Rozzi 2012). Ecológicamente, es necesario reorientar el significado del término biodiversidad hacia la noción más amplia de diversidad de vida. Éticamente, es necesario reorientar la escala de valores hacia una axiología que ubique el valor de la vida por sobre el valor del capital (Dussel 2011). Tanto la FILAC como la visión leopoldiana de la biodiversidad incluyen este sentido ético. En efecto, en nuestra cita inicial de Aldo Leopold (1949), las cuatro palabras claves son: conciencia, responsabilidad, entender y conservar.

"Conciencia" tiene dos significados, uno relacionado con la ciencia y otro con la ética. En la dimensión científica significa estar consciente o conocer los hechos. En la dimensión ética significa visualizar lo que está bien y lo que está mal. Leopold integra ambos significados del término conciencia. Si los sitios LTSER-Chile también integraran estos significados a través de la FILAC, podrían hacer aportes ecológicos y éticos para reorientar a la sociedad hacia la sustentabilidad de la vida a nivel local, nacional e internacional. De hecho, la FILAC ha comenzado a utilizarse como aproximación metodológica en la red ILTER (International Long-Term Ecological Research), particularmente en México (Aguirre 2014).

A nivel local e internacional, la integración de la conciencia ecológica y ética a través de la FILAC genera un sentido de responsabilidad que no se satisface solo con el estudio de los ecosistemas, sino que demanda relaciones de co-habitación con la comunidad de diversos seres humanos y no-humanos. Asumir este sentido de responsabilidad requiere cambios en las políticas y criterios de evaluación de la ciencia en Chile. A su vez, con estos cambios de políticas de desarrollo científico y con los aportes de una metodología que integra la investigación científica y la ética en la conciencia, la responsabilidad, el entender y la conservación, Chile puede ofrecer un cambio socio-ecológico para que el valor de la vida vuelva a estar por sobre el valor del capital en la sociedad global. Metafóricamente, podemos decir que los lentes de la red LTSER-Chile sitúan el norte de la sustentabilidad de la vida humana y no-humana en el sur del mundo.

\section{AGRADECIMIENTOS}

Agradecemos a las numerosas personas e instituciones que han colaborado con el programa de Filosofía Ambiental de Campo en el Parque Etnobotánico Omora, y al apoyo de los proyectos ICM P05-002, Basal-CONICYT PFB-23 y FONDECYT 11130451. Este trabajo es una contribución del Programa de Conservación Biocultural Subantártica (Universidad de Magallanes, Instituto de Ecología y Biodiversidad de Chile y University of North Texas).

\section{REFERENCIAS}

Acevedo M. 2006. Ecoturismo comunitario en la Ecoregión Valdiviana. In Catalán R, P Wilken, A Kandzior, D Tecklin, $\mathrm{H}$ Burschel eds. Bosque Nativo y Comunidades Locales del Sur de Chile. Santiago, Chile. Editorial Universitaria. 291$299 \mathrm{p}$.

Aguirre J. 2014. Hermeneutic and Field Environmental Philosophy to Integrate Ecological Sciences and Ethics into Earth Stewardship. In Rozzi R, FS Chapin, J B Callicott, STA Pickett, ME Power, JJ Armesto, RH May Jr. eds. Earth Stewardship: Linking ecology and ethics in theory and practice. Berlín, Alemania. Springer. p. 301-325.

Boff L. 1995. Ecology and liberation. New York, USA. Orbis Books. 187 p.

Contador TA, JH Kennedy, R Rozzi. 2014. Life cycles of freshwater invertebrates and global climate change in the subAntarctic Magellanic ecoregion: long-term ecological research at the Omora Ethonobotanical Park, Biosphere Reserve Cape Horn. Bosque 35(3): 429-437.

Contador TA, JH Kennedy, R Rozzi. 2012. The conservation status of southern South American aquatic insects in the literature. Biodiversity and Conservation 21(8): 2095-2107.

Contador TA. 2011. Benthic macroinvertebrates of temperate, sub-Antarctic streams. Tesis doctoral. Denton, EE.UU. Department of Biological Sciences, University of North Texas. 187 p.

Costanza R, R de Groot, P Sutton, S van der Ploeg, S Anderson, I Kubiszewski, S Farber. 2014. Changes in global value of ecosystem services. Global Environmental Change 26: 152-128.

Dussel E. 2011. Ética de la liberación en la edad de la globalización y de la exclusión. Madrid, España. Trotta. 664 p.

Freire P. 1970. Pedagogy of the oppressed. New York, USA. Continuum. 183 p.

Leopold A. 1949. A Sand County Almanac. New York, USA. Oxford University Press. 226 p.

Méndez M. 2013. Asociaciones Positivas de Plantas Vasculares y Musgos a Bolax gummifera (Lam.) Spreng. en Comunidades Alto-andinas de la Isla Navarino. Tesis de Magíster. Concepción, Chile. Universidad de Concepción. 91 p.

Méndez M, R Rozzi, L Cavieres. 2013. Flora vascular y no-vascular en la zona altoandina de la isla Navarino ( $\left.55^{\circ} \mathrm{S}\right)$, Reserva de biosfera Cabo de Hornos, Chile. Gayana Botanica 70: 337-343.

Mill SJ. 1848. Principios de economía política. México D.F., México. Fondo de Cultura Económica. 1157 p.

Naeem S. 2013. Ecosystem Services: Is a planet servicing one species likely to function? In Rozzi R, STA Pickett, C Palmer, JJ Armesto, JB Callicott eds. Linking ecology and ethics for a changing world. Dordrecht, Netherlands. Springer. p. 303-321.

Ojeda J. 2013. Dinámica estacional de macroalgas y moluscos intermareales en canales subantárticos del Cabo de Hornos: Una aproximación biocultural desde la filosofía ambiental de campo. Tesis de Magíster en Ciencias. Punta Arenas, Chile. Universidad de Magallanes. 144 p. 
Pisano E. 1980. Distribución y características de la vegetación del Archipiélago del Cabo de Hornos. Anales del Instituto de la Patagonia 11: 191-222.

Rozzi R. 2012. Biocultural ethics: Recovering the vital links between the inhabitants, their habits, and habitats. Environmental Ethics 43: 27-50.

Rozzi R, JE Jiménez. 2014. Magellanic subantarctic ornithology: First decade of forest bird studies at the Omora Ethnobotanical Park, Cape Horn Biosphere Reserve. Denton TX, USA - Punta Arenas, Chile. UNT Press - Ediciones Universidad de Magallanes. 364 p.

Rozzi R, JM Draguicevic, X Arango, M Sherriffs, S Ippi, C Anderson, M Acevedo, S McGehee, J Plana, E Cortés, F Massardo. 2005. Desde la ciencia hacia la conservación: el programa de educación y ética ambiental del Parque Etnobotánico Omora. Revista Ambiente y Desarrollo de CIPMA 21: 20-29.

Rozzi R, X Arango, F Massardo, C Anderson, K Heidinger, K Moses. 2008. Field environmental philosophy and biocultural conservation: the Omora Ethnobotanical Park educational program. Environmental Ethics 30: 325-336.

Rozzi R, CB Anderson, JC Pizarro, F Massardo, Y Medina, AO Mansilla, JH Kennedy, J Ojeda, V Morales, K Moses, A Poo- le, JJ Armesto, MT Kalin. 2010a. Field environmental philosophy and biocultural conservation at the Omora Ethnobotanical Park: Methodological approaches to broaden the ways of ("S") in Long-Term Socio-Ecological Research (LTSER) Sites. Revista Chilena de Historia Natural 83: 27-68.

Rozzi R, F Massardo, F Cruz, C Grenier, A Muñoz, E Mueller, J Elbers. 2010b. Galapagos and Cape Horn: ecotourism or greenwashing in two emblematic Latin American archipelagoes? Environmental Philosophy 7(2): 1-32.

Rozzi R, JJ Armesto, J Gutiérrez, F Massardo, G Likens, CB Anderson, A Poole, K Moses, G Hargrove, A Mansilla, JH Kennedy, M Willson, K Jax, C Jones, JB Callicott, MT Kalin. 2012. Integrating ecology and environmental ethics: Earth stewardship in the southern end of the Americas. BioScience 62: 226-236

Schüttler E, R Rozzi, K Jax. 2011. Towards a societal discourse on invasive species management: A case study of public perceptions of mink and beavers in Cape Horn. Journal for Nature Conservation 19: 175-184.

Smith A. 1794. Investigación de la naturaleza y causas de la riqueza de las naciones. Traducción Josef Alonso Ortiz. Valladolid, España. Oficina de la Viuda é Hijos de Santander. $1780 \mathrm{p}$.

Recibido: 10.07 .14

Aceptado: 14.10 .14 
